\title{
Medial efferent mechanisms in children with auditory processing disorders
}

\section{Srikanta K. Mishra*}

Department of Special Education and Communication Disorders, New Mexico State University, Las Cruces, NM, USA

\section{Edited by:}

Lynne E. Bernstein, George

Washington University, USA

\section{Reviewed by:}

Edward Thomas Auer, George Washington University, USA

David R. Moore, University of

Cincinnati College of Medicine, USA

\section{*Correspondence:}

Srikanta K. Mishra, Department of

Special Education and

Communication Disorders, New

Mexico State University, Las Cruces,

NM 88003, USA

e-mail:smishra@nmsu.edu
Auditory processing disorder (APD) affects about $2-5 \%$ of children. However, the nature of this disorder is poorly understood. Children with APD typically have difficulties in complex listening situations. One mechanism thought to aid in listening-in-noise is the medial olivocochlear (MOC) inhibition. The purpose of this review was to critically analyze the published data on MOC inhibition in children with APD to determine whether the MOC efferents are involved in these individuals. The otoacoustic emission (OAE) methods used to assay MOC reflex were examined in the context of the current understanding of OAE generation mechanisms. Relevant literature suggests critical differences in the study population and OAE methods. Variables currently known to influence MOC reflex measurements, for example, middle-ear muscle reflexes or OAE signal-to-noise ratio, were not controlled in most studies. The use of potentially weaker OAE methods and the remarkable heterogeneity across studies does not allow for a definite conclusion whether or not the MOC reflex is altered in children with APD. Further carefully designed studies are needed to confirm the involvement of MOC efferents in APD. Knowledge of efferent functioning in children with APD would be mechanistically and clinically beneficial.

Keywords: auditory processing disorders, medial olivocochlear reflex, otoacoustic emissions

\section{INTRODUCTION}

As many as $5 \%$ of all children with clinically normal audiograms and no obvious auditory pathology experience listening difficulty in background noise (Chermak and Musiek, 1997; Hind et al., 2011). Other researchers have reported a higher prevalence (20\%) of listening problems in children based on data from a large pediatric audiology clinic (Moore and Hunter, 2013). This condition of listening deficits despite a normal audiogram has often received a clinical label called auditory processing disorder (APD).

\section{MECHANISMS OF APD}

Despite research spanning more than two decades, the term APD is poorly defined and refers to a collection of different functional impairments (Jerger and Musiek, 2000; American SpeechLanuage-Hearing Association, 2005; American Academy of Audiology, 2010). Children with APD typically have difficulties in complex listening situations, such as understanding speech in background noise or understanding rapid or degraded speech. However, the mechanisms of APD are unclear.

The conventional hypothesis proposes that APD results from impaired "bottom-up" sensory processing and abnormal neural representation of complex acoustic signals, e.g., speech. This may

Abbreviations: APD, auditory processing disorder; ART, acoustic reflex threshold; BBN, broad-band noise; CAS, contralateral acoustic stimulation; CE, click-evoked; DPOAE, distortion product; LD, learning disability; MEMR, middle-ear muscle reflex; MOC, medial olivocochlear; OAE, otoacoustic emission; OHC, outer hair cell; SF, stimulus frequency; SLI, specific language impairment; SNR, signal-to-noise ratio; SPL, sound pressure level; TE, transient-evoked. involve lesions in the central auditory nervous system or functional impairment of basic auditory processing (Jerger and Musiek, 2000; Musiek et al., 2005; Richard, 2011); the central auditory system begins in the cochlear nucleus of the medulla and ends with the auditory cortex. However, some researchers argue that the problem may be entirely cognitive, primarily affecting attention, memory or language processing, and exerting a non-specific effect on perception (Moore, 2006, 2012; Moore et al., 2010, 2011), while others claim that APD and attentional deficits are not always related (Sharma et al., 2009). Further, there is a continuing discussion on the relationship between APD and other neurodevelopmental disorders such as specific language impairment and dyslexia (King et al., 2003; Sharma et al., 2006, 2009; Dawes et al., 2008; Ferguson et al., 2011; Moore and Hunter, 2013).

Recently, Ludwig et al. (2014) demonstrated that children with (suspected) APD with normal peripheral function [i.e., normal audiograms, stapedial reflexes, and otoacoustic emissions (OAEs)] but no associated language/reading impairments have, in fact, impaired sensory processing, manifested as a discrimination deficit in spectral and temporal domains. Their results clearly imply that APD, as a distinct clinical entity, cannot be entirely dismissed. They suggested that dichotic listening deficits in these children could be influenced by the subcortical efferent auditory system, specifically the medial olivocochlear (MOC) efferents. Accordingly, it is plausible that processing deficits expressed in the ascending auditory system could reflect the "top-down" influences from cortical centers that may exert their influence on the auditory cortex, and be conveyed to auditory periphery via the efferent pathways (Moore and Hunter, 2013). 
Another potential mechanism of speech-in-noise deficits despite a normal audiogram is hypothesized to be reduced temporal encoding precision of supra-threshold sound - termed as cochlear neuropathy (Bharadwaj et al., 2014). This can manifest both behaviorally, for example, frequency modulation discrimination, and in subcortical steady state responses in humans. Whether this is the same as APD is currently not known. Such lack of consensus in APD has affected diagnosis and management of this poorly understood disorder. For instance, one of the major issues in clinical assessment of APD is that most diagnostic tests lack clear scientific underpinnings and empirical evidence.

\section{MEDIAL OLIVOCOCHLEAR INHIBITION}

Regardless of whichever hypothesis of the pathophysiology of APD may turn out to be true, the descending system plays a significant role in hearing and auditory learning (Suga et al., 2000, 2002; Xiao and Suga, 2002; Suga and Ma, 2003; Bajo et al., 2010). The auditory system has an elaborate system of descending, efferent neural pathways that extend to the cochlea (Suga et al., 2000; Xiao and Suga, 2002). Efferent effects shape or modulate the bottom-up afferent processing. This review focuses on the medial efferents (Fex, 1962; Guinan, 2006). Auditory nerve fibers innervate reflex interneurons in the contralateral posteroventral cochlear nucleus. Axons of these interneurons cross the brainstem ventrally and innervate the medial efferent neurons on the ipsilateral side. These neurons project to the ipsilateral cochlea through the uncrossed olivocochlear bundle and modulate outer hair cell (OHC) activity (Guinan, 2006). The OHC functioning can be measured by OAEs. OAEs are sounds of cochlear origin, which can be recorded by a small microphone fitted into the ear canal. They are generated by the motion of the OHCs as they respond to auditory stimulation (Kemp, 1978).

In humans, acoustic stimulation of the MOC system results in altered cochlear amplification that is often described in terms of changes in amplitude and phase of OAEs, and is typically termed OAE suppression or MOC reflex (Guinan, 2006, 2010). In this report, the term MOC reflex is used. Animal work suggest a "MOC unmasking" hypothesis in which stimulation of the MOC efferents reduces cochlear responses to continuous noise, allowing greater responsiveness to transient signals embedded in noise (Winslow and Sachs, 1988; Kawase and Liberman, 1993; Kawase et al., 1993). While a complete understating of the role of the human MOC system in hearing is emerging (Guinan, 2006, 2010), one of the putative functions of this system is to aid in listening-in-noise (Andéol et al., 2011; de Boer et al., 2012; Abdala et al., 2014; Mishra and Lutman, 2014).

Since children with APD clinically present listening deficits in noise, a potential involvement of the MOC efferents has been investigated by some researchers as one of the underlying mechanisms of the APD (Muchnik et al., 2004; Clarke et al., 2006; Sanches and Carvallo, 2006; Garinis et al., 2008; Butler et al., 2011). The benefits of measuring MOC inhibition in children with APD are twofold. It could be useful to better define APD and identify the potential mechanisms of this disorder. Additionally, such work may elucidate the functional significance of MOC efferents in listening in complex environments during the developmental period. The potential role of the MOC system in
APD pathophysiology, should it be confirmed, would be of significant clinical interest because current APD clinical test batteries lack mechanism-based physiologic tools that are relatively quick (Emanuel, 2002; Emanuel et al., 2011). In addition, it could offer rehabilitation options for the management of APD or its subgroup through auditory training since MOC reflex strength has been shown to improve with training (Veuillet et al., 2007; de Boer and Thornton, 2008).

\section{SCOPE OF THIS REVIEW}

The objective of this review was to critically evaluate the current evidence to determine whether the MOC system is altered in individuals with APD. The literature was examined in the context of current understanding of the generation mechanism of OAEs (Shera and Guinan, 1999) and recent advancements in human MOC work (Guinan, 2006, 2010). Specifically, the included studies were analyzed for study population characteristics (how the APD was defined) and the rigor of the OAE-based MOC tests, for instance, the type of OAEs used, the quality of OAE measurements in terms of signal-to-noise ratio (SNR), techniques used to control middle-ear muscle reflexes (MEMRs), and method of computation of OAE differences.

Considering the controversies that surround APD, it is important to clarify that the intent of this review was not to precisely define APD or to agree/disagree with the concept of APD per se.

\section{MATERIALS AND METHODS}

The PubMed search engine maintained by the U.S. National Library of Medicine (http://www.ncbi.nlm.nih.gov/pubmed/) was queried for multiple specific search terms. The search period spanned more than three decades from January 1980 to April 2013. No other databases were included. The specific search terms used were (central) APD and MOC reflex, OAE suppression, or efferent inhibition. Because of the lack of a precise definition of APD, several comorbid conditions, e.g., dyslexia, learning disability (LD), listening problems, and specific language impairment were also included as search terms; however, studies on speech disorders, for example, selective mutism (Bar-Haim et al., 2004), were excluded. The following inclusion criteria were then applied: English language and human subjects. No age criteria were applied. One article written in non-English language was included as the full text was available in English. Studies were also added manually after reviewing the references of the selected journal papers.

Included studies were reviewed for study population characteristics and the rigor of the OAE-based MOC tests. Specifically, studies were examined for the type of OAEs used to assay MOC reflex, the quality of collected OAE data in terms of SNR, techniques used to control MEMRs and method of computation of OAE differences. In addition, a check was performed as to whether the included studies used state-of-the-art OAE methods, e.g., distortion product otoacoustic emission (DPOAE) fine-structure and components analysis (Abdala et al., 2009; Deeter et al., 2009; Henin et al., 2011), transient-evoked OAE wavelet analysis that retains spectral and temporal emission information (Tognola et al., 1997) or stimulus frequency OAEs (SFOAEs; Backus and Guinan, 2007) and controlled for endogenous variables such as auditory attention (Maison et al., 2001; de Boer and Thornton, 2007). 


\section{RESULTS}

Using the aforementioned search criteria, nine articles (eight on children and one on adults) were identified (Veuillet et al., 1999, 2007; Muchnik et al., 2004; Clarke et al., 2006; Sanches and Carvallo, 2006; Burguetti and Carvallo, 2008; Garinis et al., 2008; Yalçinkaya et al., 2010; Butler et al., 2011). Their principal methods and results are summarized in Table $\mathbf{1 .}$

The number of participants in the experimental groups ranged from 8 to 38 , and their auditory processing skills were typically characterized by tests recommended by the ASHA (American Speech-Lanuage-Hearing Association, 2005) or their minor variations. Eight studies used TEOAEs or click-evoked (CE) OAEs while one study used DPOAEs for assaying the MOC inhibition. The SNR of the OAE data across studies was most often $3 \mathrm{~dB}$. The MOC-induced OAE shifts were either computed by raw $\mathrm{dB}$ differences or by the equivalent attenuation method (Collet et al., 1992). Six studies used the clinical acoustic reflex procedures, but only three reported the threshold levels (Burguetti and Carvallo, 2008; Garinis et al., 2008; Butler et al., 2011). Due to these differences, a meta-analysis of literature was not feasible.

The mean MOC effect size varied from 0.6 to $2.5 \mathrm{~dB}$ across ears, frequencies, time windows, study populations, and studies. The main findings could be categorized based on three metrics: OAE level, MOC reflex magnitude, and ear asymmetry in MOC reflex. Three studies (Veuillet et al., 1999; Garinis et al., 2008; Yalçinkaya et al., 2010) reported lower OAE levels in the study group relative to the control group, while one reported relatively higher OAE levels (Muchnik et al., 2004) and the remaining five (Clarke et al., 2006; Sanches and Carvallo, 2006; Veuillet et al., 2007; Burguetti and Carvallo, 2008; Butler et al., 2011) reported no mean group differences. All but two (Clarke et al., 2006; Butler et al., 2011) studies reported that MOC reflex magnitude in the experimental group was reduced in varying degrees compared to the control group. In three (Veuillet et al., 1999, 2007; Burguetti and Carvallo, 2008) of these seven studies, the MOC reflex was lower in the right ear compared to the left ear for the experimental group in contrast to the right ear advantage in the control group. Interestingly, one study in adults with LD reported a MOC-induced increase in TEOAE level for the right ear (Garinis et al., 2008). Two reports attempted to relate the MOC reflex with performance in the behavioral tests; one study reported that the MOC reflex (magnitude and right/left asymmetry) predicted categorical perception in the APD group (Veuillet et al., 2007), while the other showed no such relationship (Muchnik et al., 2004).

None of the studies implemented state-of-the-art techniques such as DPOAE fine-structure and components unmixing, TEOAE wavelet analysis or SFOAEs to assay MOC reflex. Further attention was not considered in any of the studies.

\section{DISCUSSION}

Despite more than two decades of APD research and two decades of OAE-MOC work, only nine studies, even with a broader inclusion criterion for the study group, probed the potential malfunctioning of the MOC pathway in individuals with APD. Comparison across studies is difficult due to differences in the study populations and their auditory processing characteristics,
OAE methods and MEMR tests among the existing clinical trials. More importantly, the existing studies used relatively weak MOC test methods. These studies did not consider crucial measurement confounds necessary for valid and reliable quantification of MOC reflex (discussed later). Therefore, the present review suggests that although a MOC efferent hypothesis for modeling APD is appealing, a definitive conclusion regarding whether or not the MOC system is involved in children with APD cannot be made.

\section{MIDDLE-EAR MUSCLE REFLEXES}

One of the major concerns in OAE-based MOC studies is the possibility that the middle-ear muscle reflex contributes to the MOC reflex (or change in OAE) magnitude by influencing the stimulus and/or emissions as they transmit through the middle-ear (Guinan et al., 2003; Guinan, 2006). Depending on the level, MEMR can be evoked by contralateral broad-band noise (BBN) and/or by OAE-evoking stimulus. Included studies either did not use any MEMR test, did not report (Veuillet et al., 1999, 2007; Sanches and Carvallo, 2006) or used clinical acoustic reflex procedures (Muchnik et al., 2004; Clarke et al., 2006; Burguetti and Carvallo, 2008; Garinis et al., 2008; Yalçinkaya et al., 2010; Butler et al., 2011); see Table 1 under MEMR control. Clinical 226-Hz-probe tone procedures have been shown to yield on average 12-14 dB higher acoustic reflex thresholds, for 1000 and $2000 \mathrm{~Hz}$ activators, compared to wideband acoustic reflectance methods (Feeney et al., 2003). It is likely that MOC reflex estimation was compromised due to potential MEMR influences in studies that used high click levels to evoke OAEs (Muchnik et al., 2004; Sanches and Carvallo, 2006; Yalçinkaya et al., 2010) and/or high BBN levels to stimulate the MOC pathway (Muchnik et al., 2004; Yalçinkaya et al., 2010).

\section{OAE SIGNAL-TO-NOISE RATIO}

The noise floor associated with the OAE recording is an important variable for MOC reflex quantification because shifts in OAE level due to noise can be confused with true physiological inhibition. Likewise, lack of contralateral acoustic stimulation (CAS)-induced OAE shifts could be due to lack of inhibition or to low SNR, making the results difficult to interpret. The magnitude of the MOC effect has been reported to be dependent on the quality or SNR of the OAE data (Goodman et al., 2013). While a high SNR is desirable to detect robust MOC effects, a minimum $6 \mathrm{~dB}$ SNR has been shown to produce repeatable results (Francis and Guinan, 2010; Mishra and Lutman, 2013). Unfortunately, other than one study (Butler et al., 2011), most studies had low (3 dB) or unspecified SNR (see Table 1 under OAE protocol). Additionally, these studies did not examine potential SNR differences between the study and control groups.

\section{OAE RECORDING METHOD}

Two studies (Muchnik et al., 2004; Yalçinkaya et al., 2010) used the non-linear CEOAE recording method (Kemp et al., 1986) to assay MOC inhibition. Although the non-linear click method of recording CE/TEOAEs eliminates stimulus ringing artifacts, it cancels the linear portion of the emission, potentially removing inhibition information. In contrast, the linear click method captures the entire emission and retains complete MOC effects. 
Table 1 | Summary of the included studies.

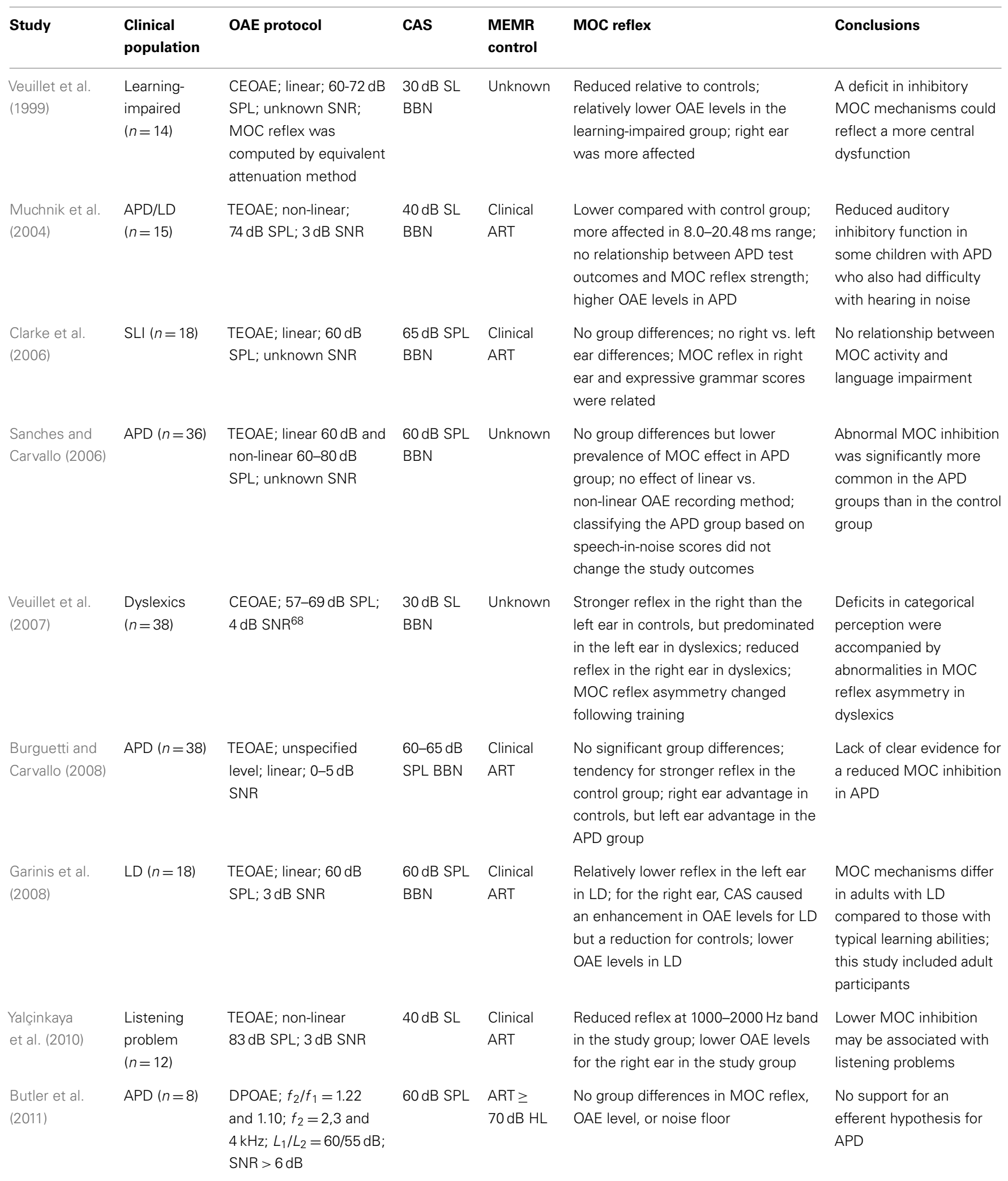

$A P D$, auditory processing disorder; ART, acoustic reflex threshold; BBN, broad-band noise; CAS, contralateral acoustic stimulation; CEOAEs, click-evoked otoacoustic emissions; DPOAEs, distortion product otoacoustic emissions; LD, learning disability; SLI, specific language impairment; SNR, signal-to-noise ratio; TEOAE, transient-evoked otoacoustic emissions. 
The DPOAE is a vector combination of at least two components, termed as distortion (generated at the overlap of primary tones $f_{1}$ and $\left.f_{2}\right)$ and reflection, produced at the $f_{d p}\left(2 f_{1}-f_{2}\right)$ place (Kim, 1980; Shera and Guinan, 1999; Talmadge et al., 1999; Knight and Kemp, 2000). The interaction of these two components in the ear canal produces oscillations in DPOAE level and phase - finestructure. The phase interference between distortion and reflection components can confound DPOAE-based measures of the MOC reflex, occasionally creating enhancements (Abdala et al., 2009; Deeter et al., 2009). Enhancements are more common at finestructure dips or minima where the components are out of phase. The MOC effect differentially influences the two components, with greater changes in the reflection component (Abdala et al., 2009, 2013; Deeter et al., 2009). The effect is not merely reduction in DPOAE level, but shifting of the fine-structure pattern mainly through the reflection component (Henin et al., 2011). Without due consideration of these physiologic mechanisms, the MOCinduced DPOAE changes may be inconclusive. For APD work, only one study used DPOAEs (Butler et al., 2011), but without any consideration to fine-structure or component separation.

\section{QUANTIFICATION OF THE MOC REFLEX}

Conventionally, the MOC reflex has been quantified by computing "raw dB differences" between OAEs with and without CAS. Most APD studies used raw dB metric to show MOC effects (Muchnik et al., 2004; Clarke et al., 2006; Sanches and Carvallo, 2006; Yalçinkaya et al., 2010; Butler et al., 2011). Such computation may not be mathematically accurate, particularly when the OAE levels between the study and control groups are different (Veuillet et al., 1999; Muchnik et al., 2004; Garinis et al., 2008; Yalçinkaya et al., 2010). Recent studies have used a variety of novel methods to compute MOC reflex (Abdala et al., 1999; Backus and Guinan, 2007; Deeter et al., 2009; Henin et al., 2011; Mishra and Lutman, 2013, 2014), for instance, a normalized index that considers the baseline OAE level or a vector index that takes into account the phase information. Three studies have specifically shown that use of normalized index provided different results or study outcomes compared to the raw dB index (Backus and Guinan, 2007; Garinis et al., 2011; Mishra and Lutman, 2014).

\section{OTHER MEASUREMENT-RELATED VARIABLES}

Other OAE- and CAS-related factors, such as slower OAE probedrifts (Henin et al., 2011; Goodman et al., 2013) and BBN bandwidths (Maison et al., 2000; Lilaonitkul and Guinan, 2009), respectively, could influence MOC reflex estimates. Lack of these specifications in published reports also makes comparison across studies difficult.

Auditory attention, a subject-related variable, has been shown to influence MOC reflex magnitude (Maison et al., 2001; de Boer and Thornton, 2007). Controlling attention by including taskdependent measures might reduce variability. This is highly relevant for comparing APD and control groups, since a well-designed, population-based study demonstrated auditory inattention as one of the underlying mechanisms of APD (Moore et al., 2010). It is plausible that the effect of auditory attention may be different in children with APD. This prediction, however, requires experimental verification.

\section{STUDY POPULATION}

An obvious challenge is the lack of consensus on what constitutes an APD. Consequently, studies have used a bewildering array of tests to recruit clinical participants that lack clear theoretical import. However, this is a generic APD research problem. Until APD is precisely defined, a working ad hoc approach could be to define the experimental group as listeners with impaired speech/listening-in-noise performance, with normal peripheral function and with typical speech-language and learning abilities. It would also be informative to test whether a relationship exists between MOC impairment and the behavioral test outcome in these children (Veuillet et al., 2007).

\section{SUMMARY}

This review suggests that the rigor of MOC reflex measurements in children with APD reported in the available literature is not at par with the recent advancements in OAE-based assays of the MOC reflex. Valid and reliable assessment of the efferent system is lacking in the extant studies.

A related outstanding question is whether MOC efferent mechanisms are important for auditory development. Current understanding of the role of the MOC efferents in auditory perception in children is sparse. Limited data exist showing the relationship between the MOC reflex and speech perception in noise in 10-12year-old (Kumar and Vanaja, 2004) and 13-17-year-old children (Abdala et al., 2014). It is expected that future studies along these lines will use state-of-the-art OAE-MOC tests that would not only help underpin the mechanisms of APD, but would also enhance the current understanding of the functional significance of the MOC system during development. Ultimately, such work may lead to clinically relevant outcomes.

\section{ACKNOWLEDGMENTS}

The author greatly appreciates and thanks John Mugge for his assistance with the preparation of this manuscript.

\section{REFERENCES}

Abdala, C., Dhar, S., Ahmadi, M., and Luo, P. (2014). Aging of the medial olivocochlear reflex and associations with speech perception. J. Acoust. Soc. Am. 135, 754-765. doi:10.1121/1.4861841

Abdala, C., Ma, E., and Sininger, Y. S. (1999). Maturation of medial efferent system function in humans. J. Acoust. Soc. Am. 105, 2392-2402. doi:10.1121/ 1.426844

Abdala, C., Mishra, S., and Garinis, A. (2013). Maturation of the human medial efferent reflex revisited. J. Acoust. Soc. Am. 133, 938-950. doi:10.1121/1.4773265

Abdala, C., Mishra, S. K., and Williams, T. L. (2009). Considering distortion product otoacoustic emission fine structure in measurements of the medial olivocochlear reflex. J. Acoust. Soc. Am. 125, 1584-1594. doi:10.1121/1.3068442

American Academy of Audiology. (2010). Diagnosis, treatment and management of children and adults with central auditory processing disorder. Clin. Pract. Guidel. 1-51. Available at: http://www.psha.org/pdfs/toolbox/ CAPD-Guidelines_8-2010.pdf

American Speech-Lanuage-Hearing Association. (2005). Central Auditory Processing Disorders. Rockville, MD: American Speech-Language-Hearing Association, 1-20. Available at: http://www.asha.org/policy/TR2005-00043/

Andéol, G., Guillaume, A., Micheyl, C., Savel, S., Pellieux, L., and Moulin, A. (2011). Auditory efferents facilitate sound localization in noise in humans. J. Neurosci. 31, 6759-6763. doi:10.1523/jneurosci.0248-11.2011

Backus, B. C., and Guinan, J. J. (2007). Measurement of the distribution of medial olivocochlear acoustic reflex strengths across normal-hearing individuals via otoacoustic emissions. J. Assoc. Res. Otolaryngol. 8, 484-496. doi:10.1007/s10162007-0100-0 
Bajo, V. M., Nodal, F. R., Moore, D. R., and King, A. J. (2010). The descending corticocollicular pathway mediates learning-induced auditory plasticity. Nat. Neurosci. 13, 253-260. doi:10.1038/nn.2466

Bar-Haim, Y., Henkin, Y., Ari-Even-Roth, D., Tetin-Schneider, S., Hildesheimer, M., and Muchnik, C. (2004). Reduced auditory efferent activity in childhood selective mutism. Biol. Psychiatry 55, 1061-1068. doi:10.1016/j.biopsych.2004.02.021

Bharadwaj, H. M., Verhulst, S., Shaheen, L., Liberman, M. C., and ShinnCunningham, B. G. (2014). Cochlear neuropathy and the coding of suprathreshold sound. Front. Syst. Neurosci. 8:26. doi:10.3389/fnsys.2014.00026

Burguetti, F. A., and Carvallo, R. M. (2008). Efferent auditory system: effect on auditory processing. Braz. J. Otorhinolaryngol. 74, 737-745.

Butler, B. E., Purcell, D. W., and Allen, P. (2011). Contralateral inhibition of distortion product otoacoustic emissions in children with auditory processing disorders. Int. J. Audiol. 50, 530-539. doi:10.3109/14992027.2011.582167

Chermak, G. D., and Musiek, F. E. (1997). Central Auditory Processing Disorders: New Perspectives. San Diego, CA: Singular Publishing Group, Inc.

Clarke, E. M., Ahmmed, A., Parker, D., and Adams, C. (2006). Contralateral suppression of otoacoustic emissions in children with specific language impairment. Ear Hear. 27, 153-160. doi:10.1097/01.aud.0000204311.73871.41

Collet, L., Veuillet, E., Bene, J., and Morgon, A. (1992). Effects of contralateral white noise on click-evoked emissions in normal and sensorineural ears: towards an exploration of the medial olivocochlear system. Audiology 31, 1-7. doi:10.3109/00206099209072897

Dawes, P., Bishop, D. V., Sirimanna, T., and Bamiou, D. E. (2008). Profile and aetiology of children diagnosed with auditory processing disorder (APD). Int. J. Pediatr. Otorhinolaryngol. 72, 483-489. doi:10.1016/j.ijporl.2007.12.007

de Boer, J., and Thornton, A. R. (2007). Effect of subject task on contralateral suppression of click evoked otoacoustic emissions. Hear. Res. 233, 117-123. doi:10.1016/j.heares.2007.08.002

de Boer, J., and Thornton, A. R. (2008). Neural correlates of perceptual learning in the auditory brainstem: efferent activity predicts and reflects improvement at a speech-in-noise discrimination task. J. Neurosci. 28, 4929-4937. doi:10.1523/jneurosci.0902-08.2008

de Boer, J., Thornton, A. R., and Krumbholz, K. (2012). What is the role of the medial olivocochlear system in speech-in-noise processing? J. Neurophysiol. 107, 1301-1312. doi:10.1152/jn.00222.2011

Deeter, R., Abel, R., Calandruccio, L., and Dhar, S. (2009). Contralateral acoustic stimulation alters the magnitude and phase of distortion product otoacoustic emissions. J. Acoust. Soc. Am. 126, 2413-2424. doi:10.1121/1.3224716

Emanuel, D. C. (2002). The auditory processing battery: survey of common practices. J. Am. Acad. Audiol. 13, 93-117; quiz 118-119.

Emanuel, D. C., Ficca, K. N., and Korczak, P. (2011). Survey of the diagnosis and management of auditory processing disorder. Am. J. Audiol. 20, 48-60. doi:10.1044/1059-0889(2011/10-0019)

Feeney, M. P., Keefe, D. H., and Marryott, L. P. (2003). Contralateral acoustic reflex thresholds for tonal activators using wideband energy reflectance and admittance. J. Speech Lang. Hear. Res. 46, 128-136. doi:10.1044/1092-4388(2003/010)

Ferguson, M. A., Hall, R. L., Riley, A., and Moore, D. R. (2011). Communication, listening, cognitive and speech perception skills in children with auditory processing disorder (APD) or specific language impairment (SLI). J. Speech Lang. Hear. Res. 54, 211-227. doi:10.1044/1092-4388(2010/09-0167)

Fex, J. (1962). Auditory activity in centrifugal and centripetal cochlear fibres in the cat. Acta Physiol. Scand. Suppl. 189, 1-68.

Francis, N., and Guinan, J. J. (2010). Acoustic stimulation of human medial olivocochlear efferents reduces stimulus frequency- and click-evoked otoacoustic emission delays: implications for cochlear filter bandwidths. Hear. Res. 267, 36-45. doi:10.1016/j.heares.2010.04.009

Garinis, A. C., Glattke, T., and Cone, B. K. (2011). The MOC reflex during active listening to speech. J. Speech Lang. Hear. Res. 54, 1464-1477. doi:10.1044/10924388(2011/10-0223)

Garinis, A. C., Glattke, T., and Cone-Wesson, B. K. (2008). TEOAE suppression in adults with learning disabilities. Int. J. Audiol. 47, 607-614. doi:10.1080/ 14992020802129402

Goodman, S. S., Mertes, I. B., Lewis, J. D., and Weissbeck, D. K. (2013). Medial olivocochlear-induced transient-evoked otoacoustic emission amplitude shifts in individual subjects. J. Assoc. Res. Otolaryngol. 14, 829-842. doi:10.1007/s10162013-0409-9
Guinan, J. J. (2006). Olivocochlear efferents: anatomy, physiology, function, and the measurement of efferent effects in humans. Ear Hear. 27, 589-607. doi:10.1097/ 01.aud.0000240507.83072.e7

Guinan, J. J. (2010). Cochlear efferent innervation and function. Curr. Opin. Otolaryngol. Head Neck Surg. 18, 447-453. doi:10.1097/MOO.0b013e32833e05d6

Guinan, J. J., Backus, B. C., Lilaonitkul, W., and Aharonson, V. (2003). Medial olivocochlear efferent reflex in humans: otoacoustic emission (OAE) measurement issues and the advantages of stimulus frequency OAEs. J. Assoc. Res. Otolaryngol. 4, 521-540. doi:10.1007/s10162-002-3037-3

Henin, S., Thompson, S., Abdelrazeq, S., and Long, G. R. (2011). Changes in amplitude and phase of distortion-product otoacoustic emission fine-structure and separated components during efferent activation. J. Acoust. Soc. Am. 129, 2068-2079. doi:10.1121/1.3543945

Hind, S. E., Haines-Bazrafshan, R., Benton, C. L., Brassington, W., Towle, B., and Moore, D. R. (2011). Prevalence of clinical referrals having hearing thresholds within normal limits. Int. J. Audiol. 50, 708-716. doi:10.3109/14992027.2011. 582049

Jerger, J., and Musiek, F. (2000). Report of the consensus conference on the diagnosis of auditory processing disorders in school-aged children. J. Am. Acad. Audiol. 11, 467-474.

Kawase, T., Delgutte, B., and Liberman, M. C. (1993). Antimasking effects of the olivocochlear reflex. II. Enhancement of auditory-nerve response to masked tones. J. Neurophysiol. 70, 2533-2549.

Kawase, T., and Liberman, M. C. (1993). Antimasking effects of the olivocochlear reflex. I. Enhancement of compound action potentials to masked tones. J. Neurophysiol. 70, 2519-2532.

Kemp, D. T. (1978). Stimulated acoustic emissions from within the human auditory system. J. Acoust. Soc. Am. 64, 1386-1391. doi:10.1121/1.382104

Kemp, D. T., Bray, P., Alexander, L., and Brown, A. M. (1986). Acoustic emission cochleography - practical aspects. Scand. Audiol. Suppl. 25, 71-95.

Kim, D. O. (1980). Cochlear mechanics: implications of electrophysiological and acoustical observations. Hear. Res. 2, 297-317. doi:10.1016/0378-5955(80) 90064-7

King, W. M., Lombardino, L. J., Crandell, C. C., and Leonard, C. M. (2003). Comorbid auditory processing disorder in developmental dyslexia. Ear Hear. 24, 448-456. doi:10.1097/01.AUD.0000090437.10978.1A

Knight, R. D., and Kemp, D. T. (2000). Indications of different distortion product otoacoustic emission mechanisms from a detailed $\mathrm{f} 1, \mathrm{f} 2$ area study. J. Acoust. Soc. Am. 107, 457-473. doi:10.1121/1.428351

Kumar, U. A., and Vanaja, C. S. (2004). Functioning of olivocochlear bundle and speech perception in noise. Ear Hear. 25, 142-146. doi:10.1097/01.AUD. 0000120363.56591.E6

Lilaonitkul, W., and Guinan, J. J. (2009). Human medial olivocochlear reflex: effects as functions of contralateral, ipsilateral, and bilateral elicitor bandwidths. J. Assoc. Res. Otolaryngol. 10, 459-470. doi:10.1007/s10162-009-0163-1

Ludwig, A. A., Fuchs, M., Kruse, E., Uhlig, B., Kotz, S. A., and Rübsamen, R. (2014). Auditory processing disorders with and without central auditory discrimination deficits. J. Assoc. Res. Otolaryngol. 15, 441-464. doi:10.1007/s10162014-0450-3

Maison, S., Micheyl, C., Andéol, G., Gallégo, S., and Collet, L. (2000). Activation of medial olivocochlear efferent system in humans: influence of stimulus bandwidth. Hear. Res. 140, 111-125. doi:10.1016/S0378-5955(99)00196-3

Maison, S., Micheyl, C., and Collet, L. (2001). Influence of focused auditory attention on cochlear activity in humans. Psychophysiology 38, 35-40. doi:10.1111/ 1469-8986.3810035

Mishra, S. K., and Lutman, M. E. (2013). Repeatability of click-evoked otoacoustic emission-based medial olivocochlear efferent assay. Ear Hear. 34, 789-798. doi:10.1097/AUD.0b013e3182944c04

Mishra, S. K., and Lutman, M. E. (2014). Top-down influences of the medial olivocochlear efferent system in speech perception in noise. PLoS ONE 9:e85756. doi:10.1371/journal.pone.0085756

Moore, D. R. (2006). Auditory processing disorder (APD)-potential contribution of mouse research. Brain Res. 1091, 200-206. doi:10.1016/j.brainres.2006. 01.108

Moore, D. R. (2012). Listening difficulties in children: bottom-up and top-down contributions. J. Commun. Disord. 45, 411-418. doi:10.1016/j.jcomdis. 2012.06.006 
Moore, D. R., Cowan, J. A., Riley, A., Edmondson-Jones, A. M., and Ferguson, M. A. (2011). Development of auditory processing in 6- to 11-yr-old children. Ear Hear. 32, 269-285. doi:10.1097/aud.0b013e318201c468

Moore, D. R., Ferguson, M. A., Edmondson-Jones, A. M., Ratib, S., and Riley, A. (2010). Nature of auditory processing disorder in children. Pediatrics 126, e382-e390. doi:10.1542/peds.2009-2826

Moore, D. R., and Hunter, L. L. (2013). Auditory processing disorder (APD) in children: a marker of neurodevelopmental syndrome. Hear. Balance Commun. 11, 160-167. doi:10.3109/21695717.2013.821756

Muchnik, C., Ari-Even Roth, D., Othman-Jebara, R., Putter-Katz, H., Shabtai, E. L., and Hildesheimer, M. (2004). Reduced medial olivocochlear bundle system function in children with auditory processing disorders. Audiol. Neurootol. 9, 107-114. doi:10.1159/000076001

Musiek, F. E., Shinn, J. B., Jirsa, R., Bamiou, D.-E., Baran, J. A., and Zaida, E. (2005). GIN (gaps-in-noise) test performance in subjects with confirmed central auditory nervous system involvement. Ear Hear. 26, 608-618. doi:10.1097/01.aud. 0000188069.80699 .41

Richard, G. J. (2011). The role of the speech-language pathologist in identifying and treating children with auditory processing disorder. Lang. Speech Hear. Serv. Sch. 42, 297-302. doi:10.1044/0161-1461(2011/10-0080)

Sanches, S. G., and Carvallo, R. M. (2006). Contralateral suppression of transient evoked otoacoustic emissions in children with auditory processing disorder. Audiol. Neurootol. 11, 366-372. doi:10.1159/000095898

Sharma, M., Purdy, S. C., and Kelly, A. S. (2009). Comorbidity of auditory processing, language, and reading disorders. J. Speech Lang. Hear. Res. 52, 706-722. doi:10.1044/1092-4388(2008/07-0226)

Sharma, M., Purdy, S. C., Newall, P., Wheldall, K., Beaman, R., and Dillon, H. (2006). Electrophysiological and behavioral evidence of auditory processing deficits in children with reading disorder. Clin. Neurophysiol. 117, 1130-1144. doi:10.1016/j.clinph.2006.02.001

Shera, C. A., and Guinan, J. J. (1999). Evoked otoacoustic emissions arise by two fundamentally different mechanisms: a taxonomy for mammalian OAEs. J. Acoust. Soc. Am. 105, 782-798. doi:10.1121/1.426948

Suga, N., Gao, E., Zhang, Y., Ma, X., and Olsen, J. F. (2000). The corticofugal system for hearing: recent progress. Proc. Natl. Acad. Sci. U.S.A. 97, 11807-11814. doi:10.1073/pnas.97.22.11807

Suga, N., and Ma, X. (2003). Multiparametric corticofugal modulation and plasticity in the auditory system. Nat. Rev. Neurosci. 4, 783-794. doi:10.1038/ nrn 1222
Suga, N., Xiao, Z., Ma, X., and Ji, W. (2002). Plasticity and corticofugal modulation for hearing in adult animals. Neuron 36, 9-18. doi:10.1016/S0896-6273(02) 00933-9

Talmadge, C. L., Long, G. R., Tubis, A., and Dhar, S. (1999). Experimental confirmation of the two-source interference model for the fine structure of distortion product otoacoustic emissions. J. Acoust. Soc. Am. 105, 275-292. doi:10.1121/1.424584

Tognola, G., Grandori, F., and Ravazzani, P. (1997). Time-frequency distributions of click-evoked otoacoustic emissions. Hear. Res. 106, 112-122. doi:10.1016/S03785955(97)00007-5

Veuillet, E., Collet, L., and Bazin, F. (1999). Objective evidence of peripheral auditory disorders in learning-impaired children. J. Audiol. Med. 8, 18-29.

Veuillet, E., Magnan, A., Ecalle, J., Thai-Van, H., and Collet, L. (2007). Auditory processing disorder in children with reading disabilities: effect of audiovisual training. Brain 130, 2915-2928. doi:10.1093/brain/awm235

Winslow, R. L., and Sachs, M. B. (1988). Single-tone intensity discrimination based on auditory-nerve rate responses in backgrounds of quiet, noise, and with stimulation of the crossed olivocochlear bundle. Hear. Res. 35, 165-189. doi:10.1016/0378-5955(88)90116-5

Xiao, Z., and Suga, N. (2002). Modulation of cochlear hair cells by the auditory cortex in the mustached bat. Nat. Neurosci. 5, 57-63. doi:10.1038/nn786

Yalçinkaya, F., Yilmaz, S. T., and Muluk, N. B. (2010). Transient evoked otoacoustic emissions and contralateral suppressions in children with auditory listening problems. Auris Nasus Larynx 37, 47-54. doi:10.1016/j.anl.2009.02.010

Conflict of Interest Statement: The author declares that the research was conducted in the absence of any commercial or financial relationships that could be construed as a potential conflict of interest.

Received: 26 June 2014; accepted: 06 October 2014; published online: 27 October 2014 Citation: Mishra SK (2014) Medial efferent mechanisms in children with auditory processing disorders. Front. Hum. Neurosci. 8:860. doi: 10.3389/fnhum.2014.00860 This article was submitted to the journal Frontiers in Human Neuroscience.

Copyright (C) 2014 Mishra. This is an open-access article distributed under the terms of the Creative Commons Attribution License (CC BY). The use, distribution or reproduction in other forums is permitted, provided the original author(s) or licensor are credited and that the original publication in this journal is cited, in accordance with accepted academic practice. No use, distribution or reproduction is permitted which does not comply with these terms. 